\title{
Uso de hábitat por aves en rellenos sanitarios del noreste de la provincia de Buenos Aires, Argentina
}

\author{
Germán Marateo ${ }^{凶}$; Pablo Grilli; Nancy Bouzas; Roberto Jensen; Vanina \\ Ferretti; Marcos JuÁrez \& Guillermo SoAve
}

Facultad de Ciencias Naturales y Museo, Universidad Nacional de La Plata, La Plata, Argentina

\begin{abstract}
RESUMEN. Los basurales a cielo abierto y los rellenos sanitarios son sitios de atracción para diferentes especies de aves oportunistas que pueden aumentar sus poblaciones debido al suplemento alimentario brindado por los residuos orgánicos. Estas aves pueden actuar como vectores de enfermedades o contaminantes, causar daños a la agricultura, aumentar el riesgo de accidentes aéreos y tener efectos negativos sobre otras especies por competencia o depredación. Los objetivos de este trabajo son identificar y cuantificar las especies de aves y el uso de hábitat en tres rellenos sanitarios del noreste de la provincia de Buenos Aires y determinar la importancia de la cantidad de residuos depositados sobre dichas variables durante dos períodos invernales consecutivos. La abundancia total de aves fue diferente entre los rellenos en ambos períodos de estudio, aunque las gaviotas fue siempre el grupo más abundante en los tres rellenos. El relleno Norte 3 tuvo la mayor abundancia de gaviotas y palomas. Las aves zancudas fueron significativamente más abundantes en el relleno de González Catán, y las rapaces tuvieron abundancias similares en los tres rellenos en ambos períodos. En ambos períodos las gaviotas de los tres rellenos fueron abundantes en todos los ambientes, mientras que sólo la abundancia de las palomas en el relleno Norte 3 estuvo relacionada con las celdas de disposición final y el borde cercano. Se encontró relación entre la superficie de residuos expuestos y la abundancia total de aves y la abundancia de palomas, que a su vez se relacionó con la cantidad diaria de residuos depositados. La reducción de la porción orgánica y el manejo de los residuos durante la disposición final, el manejo de los ambientes aledaños, junto a métodos directos pasivos y activos de control, reducirían las abundancias de aves y la potencialidad de los efectos negativos para el hombre y el ambiente.
\end{abstract}

[Palabras clave: residuos, gaviotas, palomas, ambientes disturbados, antropización y avifauna, rellenos sanitarios]

\begin{abstract}
Aвstract. Habitat use by birds in landfills of northeastern Buenos Aires province, Argentina: Rubbish dumps and landfills are attractive sites for different species of opportunistic birds which can increase their populations on the food supplement offered by organic waste. These birds can act like vectors of diseases or polluting agents, damage agriculture, increase the risk of bird strikes, and have negative effects on other species through competition or predation. The goals of this work are to identify and quantify the species of birds and their habitat use within three landfills of the northeast of the Buenos Aires province, and to determine the importance of the waste amount deposited on these variables during two consecutive winter periods. Total bird abundance was different between landfills in both winters, and gulls predominated in the three landfills. North 3 landfill had the greater abundance of gulls and doves. Wading birds were significantly more abundant in González Catán Landfill, and the birds of prey had similar abundances in the three landfills in both periods. In both periods, the gulls were abundant in all habitats of three landfills, whereas only the abundance of doves in North 3 landfill was related to the final waste disposition sites and to the near edge $(<200 \mathrm{~m})$ area. Exposed waste in surface was positively correlated with total bird abundance and the abundance of doves, which are themselves related to the daily amount waste deposited. Reduction of the organic portion and waste handling during the final disposition, habitat management around the final disposition sites, together with both passive and active direct control methods would reduce the abundance of birds in landfills and their negative potential effects on man and the environment.
\end{abstract}

[Keywords: waste, gulls, doves, environment disturbed, man and birds, landfills]

\section{INTRODUCCIÓN}

El crecimiento poblacional humano conlleva un incremento de la generación de residuos sólidos urbanos (Hunt 1972; Blokpoel \& Spaans 1991). Los residuos requieren de un sistema de gestión que contempla su almacenamiento en origen, su recolección, transferencia y disposición final. En Argentina, los residuos de origen domiciliario son depositados de dos formas: basurales a cielo

Editor asociado: Fernando Milesi

$\triangle$ gmarateo@yahoo.com abierto y rellenos sanitarios. El relleno sanitario es un método de disposición final de residuos sólidos y semisólidos que confina los residuos en la menor superficie posible; los residuos son reducidos a un volumen mínimo y cubiertos con tierra (ASCE 1969).

Estos sitios constituyen a menudo puntos atractivos para distintas especies de aves, que los utilizan como fuentes alternativas o 
principales de alimentación (Pomeroy 1975; Donázar 1992; Belant 1997). El aumento en cantidad y la distribución de muchas poblaciones de gaviotas en diversos lugares del mundo han sido atribuidos con frecuencia al suplemento alimentario de origen antrópico existente en estos sitios (e.g., Kadlec \& Drury 1968; Harris 1970; Drury 1973; Conover 1983; Patton 1988; Spaans \& Blokpoel 1991; Belant \& Dolbeer 1993; Belant et al. 1993) y Argentina no escapa a dicho patrón (Yorio et al. 2005). Las aves que usan los vertederos pueden actuar como vectores de enfermedades o contaminantes, y cuando sus abundancias son altas pueden causar daños a la agricultura, aumentar el riesgo de colisiones con aviones ocasionando accidentes aéreos y tener efectos negativos sobre otras especies por competencia o depredación (Butterfield et al. 1983; Monaghan et al. 1985; Blokpoel \& Spaans 1991; Sodhi 2002).

La disposición de residuos sin tratamiento previo está asociada a congregaciones mayores de aves respecto de aquellos lugares en los cuales los residuos son incinerados o compactados (Patton 1988; Spaans \& Blokpoel 1991; Pons 1992; Giaccardi et al. 1997). Aún en los rellenos sanitarios, la cantidad de residuos expuestos antes de ser cubiertos por completo podría influir en la abundancia de aves que los utilizan. Además, los ambientes que rodean a los rellenos sanitarios también pueden determinar la presencia y abundancia de determinadas especies de aves. El manejo adecuado de los residuos y de los ambientes que rodean a los sitios de disposición final cobra vital importancia a la hora de realizar estrategias de control y manejo de las aves que los usan.

Los estudios de aves y vertederos se encuentran mejor desarrollados en Europa, América del Norte, África y Australia; en Sudamérica son escasos. Existen unos pocos estudios en Argentina que evaluaron la utilización de basurales a cielo abierto y desechos pesqueros, principalmente por especies de gaviotas (familia Laridae) en Patagonia (Yorio et al. 1996; Giaccardi et al. 1997; Bertellotti et al. 2001; Yorio \& Giaccardi 2002; Giaccardi \& Yorio 2004). Sin embargo, hasta el presente no se cuenta con estudios en rellenos sanitarios. Los objetivos de este trabajo son identificar y cuantificar las especies de aves que utilizan los tres rellenos sanitarios de CEAMSE ubicados en el noreste de la provincia de Buenos Aires, evaluar el uso del espacio que realizan las aves dentro de ellos, y determinar la importancia de la superficie de residuos expuestos y la cantidad de residuos depositados en la abundancia y el uso de hábitat de dichas especies.

\section{Métodos}

\section{Área de estudio}

El estudio se realizó en tres rellenos sanitarios que reciben residuos domiciliarios y asimilables y son operados por CEAMSE (Coordinación Ecológica Ârea Metropolitana Sociedad del Estado).

Los rellenos estudiados, González Catán (34²47' S, $\left.58^{\circ} 40^{\prime} \mathrm{W}\right)$, Norte 3 ( $\left.34^{\circ} 31^{\prime} \mathrm{S}, 58^{\circ} 36^{\prime} \mathrm{W}\right)$ y Ensenada (34 $\left.51^{\prime} \mathrm{S}, 5^{\circ} 57^{\prime} \mathrm{W}\right)$, se encuentran ubicados en los partidos de La Matanza, San Miguel y Ensenada, respectivamente, en el noreste de la provincia de Buenos Aires. Durante el periodo de estudio, las toneladas de residuos depositadas mensualmente fueron de aproximadamente 41500, 384490, 28250 respectivamente (CEAMSE, comunicación pública). Los residuos provienen de la Ciudad Autónoma de Buenos Aires, de la ciudad de La Plata, Berisso y Ensenada, y de los partidos aledaños, denominados comúnmente como Gran Buenos Aires y Gran La Plata. Los residuos orgánicos conforman casi el $40 \%$ del total de residuos depositados (CEAMSE, comunicación pública).

El clima de la región es templado húmedo sin estación seca, con una temperatura media anual de $17^{\circ} \mathrm{C}$, una precipitación anual que oscina entre 1000 y 1200 mm y una evapotranspiración potencial de entre 850 y $900 \mathrm{~mm} /$ año, por lo que siempre existe un exceso hídrico. En la zona de la ribera del Río de la Plata el microclima es menos riguroso, con temperaturas extremas menos pronunciadas y menor frecuencia e intensidad de heladas (Servicio Meteorológico Nacional, comunicación pública).

Muchas de las comunidades vegetales naturales de la zona han sido reemplazadas, modificadas o restringidas en su distribución por encontrarse en una zona en franca expansión demográfica y urbanización. La vegetación natural ha sido casi totalmente removida, empobrecida o sustituida por especies de cultivo, o los campos han sido muy alterados por las prácticas agroganaderas o por el desarrollo urbano. A su vez, los bosques naturales han sido alterados en mayor o menor medida por tala, retracción y aislamiento, plantación e invasión de especies exóticas (Brown et al. 2006).

El relleno de Gonzalez Catán está ubicado en un espacio abierto y flanqueado por el Arroyo Morales y dos arroyos afluentes. La vegetación de la zona es principalmente herbácea, aunque existen pequeñas arboledas. En el relleno Norte 3 existen zonas abiertas, humedales, una laguna de origen antrópico, áreas arboladas, y se encuentra flanqueado por el río Reconquista. La cercanía del aeródromo del Comando de Aviación del Ejército de la Guarnición Militar de Campo de Mayo $(\sim 4 \mathrm{~km})$, hace de este relleno un sitio de especial importancia en relación al manejo de las especies 
de aves asociadas, dado que su presencia representa un riesgo para las operaciones de las aeronaves. En el área del relleno Ensenada existen dos lagunas de origen antrópico. Una de ellas dentro de los límites del predio y la otra, laguna Los Patos, $250 \mathrm{~m}$ fuera del predio. Además en la zona hay pastizales, pajonales, campos inundables, bañados y canales arbolados.

\section{Muestreo}

El muestreo de aves se hizo durante los períodos otoño/invernales de 2007 (mayo a agosto) y 2008 (abril a septiembre). El período otoño/invernal es el de mayor abundancia de aves en los tres rellenos estudiados (datos no publicados).

Se realizaron 33 muestreos (18 en 2007 y 15 en 2008) en los tres rellenos, a intervalos entre 10 y 40 días. En cada muestreo se determinaron la abundancia de cada especie (número de individuos), el uso del hábitat de cada especie (número de individuos de cada especie por ambiente) y el tamaño del frente expuesto de disposición de residuos (superficie de residuos expuestos). Cada muestreo fue realizado por dos observadores por la mañana (entre las 10 y las $12 \mathrm{~h}$ ), quienes recorrieron de manera sistemática los bordes de cada área de disposición de residuos, deteniéndose a intervalos regulares para localizar, identificar y contar (o estimar en caso de las especies más abundantes) todos los individuos en los distintos ambientes seleccionados. Las estimaciones de abundancia de las especies más abundantes fueron realizadas mediante conteos parciales de bandadas de entre 100 y 500 individuos, extrapolando al total de la población presente por sectores.

Dentro de los rellenos se determinaron cuatro ambientes: 1) celdas de disposición final (CDF): áreas donde quedan residuos expuestos y que son cubiertos constantemente con áridos; 2) borde cercano (BC): perímetro lindero inmediato a los CDF hasta un radio de $200 \mathrm{~m}$ de distancia, con una gran proporción de superficie de suelo desnudo debido al tapado reciente de residuos con áridos, y en menor proporción de suelos con cobertura de estrato herbáceo bajo ( $\leq 20 \mathrm{~cm}$ de altura); 3 ) borde lejano (BL): perímetro más alejado de los CDF (>200 $\mathrm{m}$ de distancia), generalmente con suelos cubiertos por un estrato herbáceo ( $>20 \mathrm{~cm}$ de altura) y arboledas, pajonales o pastizales inundables en menor proporción; y 4) ambientes acuáticos (Amb. ac.): lagunas naturales o artificiales.

\section{Análisis de datos}

Se analizaron sólo las abundancias de las especies de aves no Passeriformes por representar más de 95\% de la abundancia total. Las especies de aves que se registraron utilizando las áreas de residuos expuestos fueron agrupadas en cuatro grupos funcionales mayores: 1) gaviotas (Gaviota Cocinera Larus dominicanus, Lichtenstein 1823, Gaviota Capucho Café Chroicocephalus maculipennis, Lichtenstein 1823; Gaviota Capucho Gris C. cirrocephalus, Vieillot 1818); 2) palomas
(Paloma Doméstica Columba livia, Gmelin 1789; Paloma Picazuró Patagioenas picazuro, Temminck 1813; Paloma Torcaza Zenaida auriculata, Des Murs 1847); 3) rapaces (Carancho Caracara plancus, Miller 1777; Chimango Milvago chimango, Vieillot 1816); y 4) zancudas (Cuervillo de Cañada Plegadis chii, Vieillot 1817; Tero Común Vanellus chilensis, Molina 1782; Tero Real Himantopus mexicanus, Statius Müller 1776; Garcita Bueyera Bubulcus ibis, Linnaeus 1758; Chiflón Syrigma sibilatrix, Temminck 1824; Cigüeña Ciconia maguari, Gmelin 1789).

Las diferencias de la abundancia total y de las abundancias de los cuatro grupos funcionales de aves entre rellenos se analizó para cada período otoño/invernal por medio de análisis de varianza no paramétricos a dos vías de clasificación o de medidas repetidas de Friedman. Se realizaron comparaciones múltiples "a posteriori" basadas en las medias de los rangos por tratamiento y en la varianza de los rangos (Conover 1999).

Para evaluar la segregación espacial delos diferentes grupos funcionales de aves de acuerdo al uso de cada ambiente dentro de los rellenos se realizaron análisis de ordenamiento multivariado para cada año de estudio. Los ordenamientos se realizaron por medio de análisis de componentes principales (ACP). Para los ACP se aplicó una transformación de raíz cuadrada para reducir las diferencias entre varianzas, y se realizaron los análisis a partir de las matrices de varianza-covarianza.

Se realizaron análisis de regresión simple entre el tamaño del frente expuesto de residuos y la abundancia de aves presentes incluyendo los datos de los tres rellenos sanitarios y los dos períodos de estudio, tomando a la superficie de residuos expuestos como variable independiente y a la abundancia total y de cada uno de los cuatro grupos funcionales de aves como variables respuesta, transformadas a escala logarítmica $(\ln +1)$. Dado que se trata de comparaciones múltiples con el mismo set de datos, se realizó una corrección del nivel de significación con protección de Bonferroni $(\alpha=0.05 / 5=0.01)$. La normalidad de la distribución de los residuos de los análisis de regresión y la homogeneidad de varianzas se analizaron gráficamente y mediante pruebas estadísticas formales (prueba de Shapiro-Wilks para poner a prueba la normalidad y de Levene para la homogeneidad de varianzas (Quinn \& Keough 2002). En este estudio, el tamaño del frente expuesto de los rellenos está directamente relacionado con la cantidad de residuos depositados en ellos (observación personal), por lo que la relación entre esa variable y la abundancia de aves implica una relación del mismo signo con la cantidad de residuos que se depositan.

\section{RESULTADOS}

La abundancia total de aves difirió significativamente entre sitios en ambos períodos de estudio. En 2008 hubo diferencias significativas entre los 3 sitios, mientras que en 
Tabla 1. Abundancia promedio $( \pm \mathrm{DE})$ de aves para dos temporadas otoño/invernales consecutivas en tres rellenos sanitarios del noreste de la provincia de Buenos Aires (Norte 3, González Catán y Ensenada). P: probabilidad asociada a la hipótesis nula según la prueba de Friedman. NS: no significativo $(P>0.05)$. Letras distintas indican diferencias significativas $(P<0.05)$ entre sitios mediante comparaciones múltiples "a posteriori" (ver texto para detalles).

Table 1. Mean $( \pm \mathrm{SD})$ abundance of birds for two consecutive autumn/winter seasons in three landfills of northeast of Buenos Aires province, Argentina.

\begin{tabular}{|c|c|c|c|c|c|c|c|c|}
\hline \multirow[b]{3}{*}{ Gaviotas } & \multicolumn{4}{|c|}{ Otoño/invierno $2007(\mathrm{n}=6)$} & \multicolumn{4}{|c|}{ Otoño/invierno $2008(n=5)$} \\
\hline & Norte 3 & Ensenada & $\begin{array}{c}\text { González } \\
\text { Catán }\end{array}$ & $\mathrm{P}$ & Norte 3 & Ensenada & $\begin{array}{c}\text { González } \\
\text { Catán }\end{array}$ & $\mathrm{P}$ \\
\hline & $\begin{array}{c}4388 \\
( \pm 4986)\end{array}$ & $\begin{array}{c}1532 \\
( \pm 1003)\end{array}$ & $\begin{array}{c}1631 \\
( \pm 1922)\end{array}$ & ns & $\begin{array}{c}12025^{\mathrm{a}} \\
( \pm 6114)\end{array}$ & $\begin{array}{l}4951^{\mathrm{a}} \\
( \pm 843)\end{array}$ & $\begin{array}{l}2019^{\mathrm{b}} \\
( \pm 422)\end{array}$ & 0.0007 \\
\hline Palomas & $\begin{array}{c}10683^{a} \\
( \pm 3980)\end{array}$ & $\begin{array}{c}553^{\mathrm{b}} \\
( \pm 212)\end{array}$ & $\begin{array}{c}568^{\mathrm{b}} \\
( \pm 308)\end{array}$ & 0.0005 & $\begin{array}{c}6883^{a} \\
( \pm 805)\end{array}$ & $\begin{array}{c}606^{\mathrm{b}} \\
( \pm 316)\end{array}$ & $\begin{array}{c}210^{c} \\
( \pm 184)\end{array}$ & $<0.0001$ \\
\hline Rapaces & $\begin{array}{c}398 \\
( \pm 211)\end{array}$ & $\begin{array}{c}259 \\
( \pm 97)\end{array}$ & $\begin{array}{c}294 \\
( \pm 79)\end{array}$ & ns & $\begin{array}{c}433 \\
( \pm 337)\end{array}$ & $\begin{array}{c}353 \\
( \pm 110)\end{array}$ & $\begin{array}{c}434 \\
( \pm 192)\end{array}$ & ns \\
\hline Zancudas & $\begin{array}{c}18^{\mathrm{a}} \\
( \pm 10)\end{array}$ & $\begin{array}{c}41^{\mathrm{a}} \\
( \pm 43)\end{array}$ & $\begin{array}{c}645^{\mathrm{b}} \\
( \pm 343)\end{array}$ & 0.0004 & $\begin{array}{l}132^{\mathrm{a}} \\
( \pm 98)\end{array}$ & $\begin{array}{c}31^{\mathrm{a}} \\
( \pm 20)\end{array}$ & $\begin{array}{c}610^{\mathrm{b}} \\
( \pm 348)\end{array}$ & 0.0007 \\
\hline Total & $\begin{array}{c}15489^{a} \\
( \pm 7047)\end{array}$ & $\begin{array}{c}2384^{\mathrm{b}} \\
( \pm 1263)\end{array}$ & $\begin{array}{c}3138^{\mathrm{b}} \\
( \pm 1845)\end{array}$ & 0.001 & $\begin{array}{c}19481^{\mathrm{a}} \\
( \pm 5923)\end{array}$ & $\begin{array}{c}5941^{\mathrm{b}} \\
( \pm 1086)\end{array}$ & $\begin{array}{l}3275^{c} \\
( \pm 865)\end{array}$ & $<0.0001$ \\
\hline
\end{tabular}

2007el rellenoNorte3 presentóuna abundancia significativamente mayor al resto. Este relleno presentó, a su vez, la mayor abundancia total para ambos períodos, mientras que el relleno de González Catán tuvo una abundancia total de aves similar en ambos períodos de estudio, y el relleno Ensenada tuvo una abundancia promedio mayor en 2008 (Tabla 1).

El relleno Norte 3 tuvo la mayor abundancia de gaviotas y palomas. Estas últimas fueron significativamente más abundantes en ambos períodos, y fueron también significativamente más abundantes en Ensenada que en González Catán en 2008, donde las gaviotas también presentaron las menores abundancias ese año. Las zancudas fueron significativamente más abundantes en González Catán en ambos períodos, mientras que las rapaces tuvieron abundancias similares en los tres sitios en ambos períodos (Tabla 1).

El análisis entre años reveló que las gaviotas fueron más abundantes en el segundo período otoño/invernal en los tres sitios, mientras que las palomas fueron más abundantes en el primero en Norte 3 y González Catán y se mantuvieron relativamente constantes en Ensenada. Las rapaces fueron más abundantes en 2008 en Ensenada y en González Catán, y se mantuvieron relativamente constantes en Norte 3. Las aves zancudas sólo aumentaron en este relleno durante 2008, siendo relativamente constantes en los otros dos sitios, aunque en Ensenada fueron poco abundantes (Tabla 1).

Para ambos años de estudio, los dos primeros ejes de los ACP explicaron más del $97 \%$ de la variabilidad total $(88.7 \%$ el eje 1 y $8.6 \%$ el eje 2 ; y $82.1 \%$ el eje 1 y $15.5 \%$ el eje 2, para los años 2007 y 2008, respectivamente). En ambos períodos, las gaviotas de los tres rellenos se relacionaron de forma positiva con todos los ambientes (eje 1), mientras que sólo la abundancia de las palomas en Norte 3 se relacionó con las celdas de disposición final

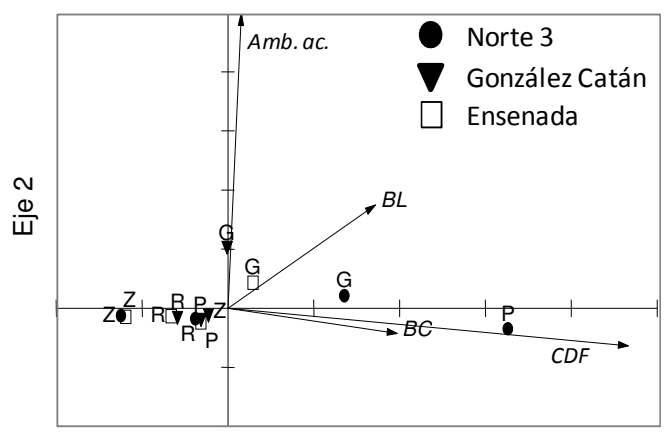

Eje 1

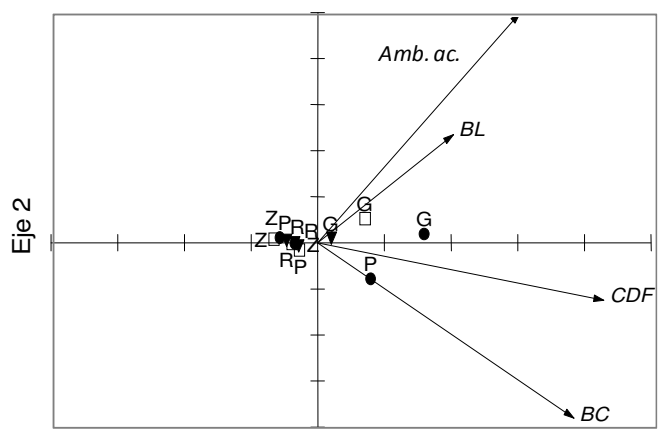

Eje 1

Figura 1. Representación gráfica de los dos primeros ejes de los ACP de los grupos funcionales de aves y los ambientes utilizados en tres rellenos sanitarios del noreste de la provincia de Buenos Aires (Norte 3, González Catán y Ensenada) durante dos temporadas otoño/invernales consecutivas: 2007 (arriba) y 2008 (abajo). Ambientes: Amb. ac.: ambientes acuáticos, BC: borde cercano, BL: borde lejano, CDF: celda de disposición final de residuos. Grupos funcionales: $\mathrm{G}=$ gaviotas, P: palomas, R: rapaces, Z: zancudas.

Figure 1. Graphical representation of both first PCA axes of the functional bird groups and the habitat used in three landfills studied, during two consecutive autumn/winter seasons. Details in Spanish legend. 
de residuos y bordes cercanos (Figura 1). En el eje 2, las gaviotas principalmente de González Catán y Ensenada tendieron a asociarse de manera positiva con los ambientes acuáticos (Figura 1). Ni el grupo de las rapaces ni el de las zancudas se relacionaron de manera relevante con los ambientes analizados, aunque las zancudas tendieron a asociarse de forma más positiva con las celdas de disposición y los bordes cercanos de González Catán (Figura 1).

Se encontró una relación significativa entre la superficie de residuos expuestos y la abundancia total de aves y la abundancia de palomas, aunque las regresiones explicaron una baja proporción de la variación (37\% y $43 \%$, respectivamente). La abundancia del resto de los grupos no se relacionó significativamente con la superficie de residuos expuestos (Tabla 2, Figura 2).

Tabla 2. Resumen de los análisis de regresión simple entre las abundancias total y de los principales grupos funcionales de aves (variables dependientes) y la superficie de residuos expuestos en tres rellenos sanitarios del noreste de la provincia de Buenos Aires, Argentina ( $\mathrm{n}=33$ muestras). ${ }^{*} P<0.01$.

Table 2. Summary of simple regression analyses between total abundance and main functional bird groups and exposed waste surface in three landfills of northeast of Buenos Aires province, Argentina.

\begin{tabular}{lcccc}
\hline Abundancia $(\mathrm{ln})$ & $\mathrm{r}^{2}$ & Coef. & $\mathrm{F}$ & $\mathrm{P}$ \\
\hline aves totales & 0.37 & $3.7 \mathrm{E}-05$ & 18.03 & $0.0002^{*}$ \\
palomas & 0.43 & $6.3 \mathrm{E}-05$ & 22.32 & $0.0001^{*}$ \\
rapaces & 0.14 & $1.1 \mathrm{E}-05$ & 4.71 & 0.04 \\
gaviotas & 0.08 & $1.7 \mathrm{E}-05$ & 2.4 & 0.1 \\
zancudas & 0.06 & $2.4 \mathrm{E}-05$ & 2.02 & 0.2 \\
\hline
\end{tabular}

\section{DiSCUSIÓN}

Las especies de aves que utilizan vertederos son oportunistas que se adaptan muy bien a ambientes antropizados (Pomeroy 1975; Donázar 1992; Belant 1997). Los residuos ofrecen una rica y variada fuente de alimentación para las especies tolerantes a esas condiciones (Pomeroy 1975; Donázar 1992; Blanco 1997; Yorio \& Giaccardi 2002).

Las gaviotas fueron el grupo predominante en los rellenos estudiados, mientras que las palomas fueron un grupo importante en abundancia en uno de ellos. La Gaviota Cocinera es la especie más abundante en los basurales de Patagonia (Yorio et al. 1996, 2005; Giaccardi et al. 1997; Yorio \& Giaccardi 2002) mientras que en los rellenos sanitarios de este estudio las especies predominantes fueron la Gaviota Capucho Café y la Gaviota Capucho Gris ( $98 \%$ de la abundancia total de gaviotas). En particular, la Gaviota Capucho
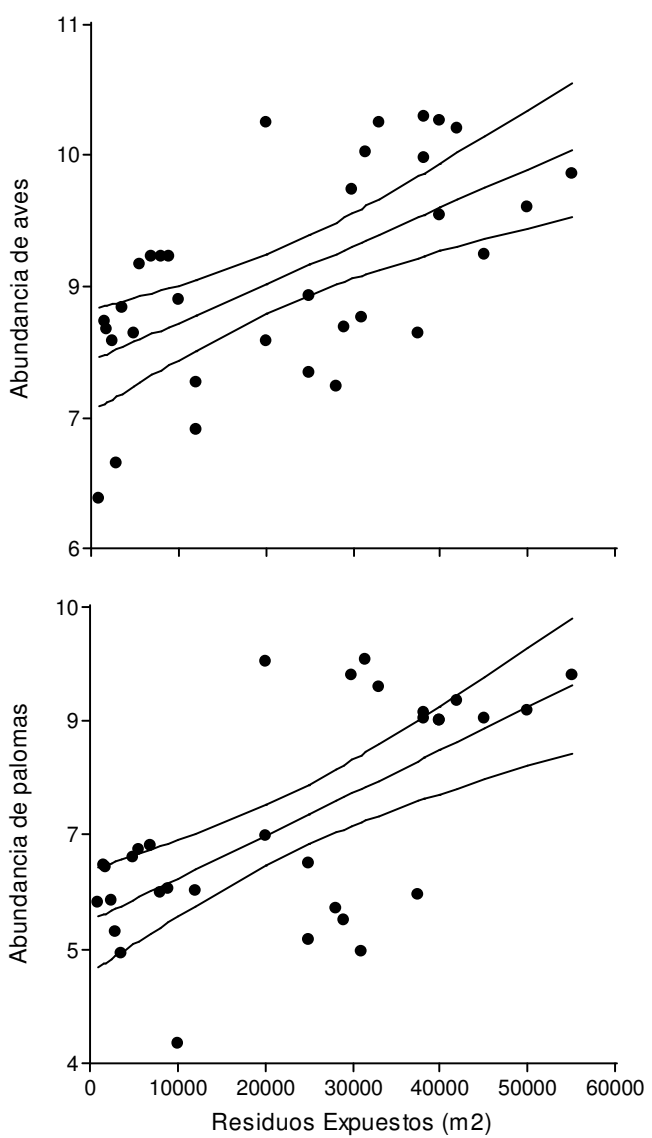

Figura 2. Línea de tendencia y límites de confianza de la regresión entre la superficie de residuos expuestos y las abundancias total de aves y del número de palomas (transformadas con logaritmos).

Figure 2. Regression tendency line and confidence limits between exposed waste surface and $\mathrm{nl}$ of the total bird numbers and the $\mathrm{nl}$ of the dove numbers.

Gris ha aumentado de manera considerable su abundancia en esta región en la última década; pasó de ser una especie escasa (Blanco \& Carbonell 2001; López-Lanús \& Blanco 2005) y de hábitos solitarios o de pequeñas bandadas en la provincia de Buenos Aires (Canevari et al. 1992; Narosky \& Di Giácomo 1993; Bó et al. 1995) a muy abundante (Blanco et al. 2008). Además, se la consideraba una especie de hábitos principalmente marinos y menos generalista que la Gaviota Capucho Café (Silva Rodríguez et al. 2005), algo que no concuerda con las elevadas abundancias halladas en este estudio. El incremento en la abundancia de esta especie probablemente esté relacionado a la gran cantidad de recursos de origen antrópico que ofrece, entre otros, la forma de tratamiento actual de los residuos. Las gaviotas parecen depender de manera muy marcada del alimento que ofrecen los residuos durante el período invernal, momento en el que las fuentes naturales de nutrientes pueden 
escasear (Kihlman \& Larsson 1974; Horton et al. 1983). Además, en esa época del año se adicionan los juveniles de la temporada reproductiva anterior, por lo que la demanda de alimento aumentaría notablemente en concordancia con las densidades poblacionales. En el período estival las gaviotas son muy poco abundantes en los rellenos estudiados (datos no publicados), retirándose probablemente hacia los sitios de nidificación.

Las aves rapaces también utilizan basurales y rellenos sanitarios en todo el mundo (Pomeroy 1975; Donázar 1992; Blanco 1994, 1997) como fuente de carroña (Houston 1994; White et al. 1994).Estegrupotieneimportantesabundancias en algunos basurales de Patagonia y Chile central (Yorio \& Giaccardi 2002; Lobos et al. 2011). En Patagonia se encontraron diferencias de abundancia de rapaces entre sitios (Yorio \& Giaccardi 2002), mientras que en el presente trabajo no tuvieron diferentes abundancias entre los tres rellenos estudiados, siendo mucho menos abundantes que las gaviotas, y generalmente también menos abundantes que las palomas. El grupo estuvo representado principalmente por chimangos y caranchos, especies oportunistas y asociadas a ambientes altamente antropizados. En Chile, el Chimango también fue la especie más abundante de rapaces de un relleno (Lobos et al. 2011).

El grupo de aves zancudas fue importante en uno de los rellenos, formado principalmente por el Cuervillo de Cañada y la Garcita Bueyera. Esta última especie fue también importante en un relleno de Chile central (Lobos et al. 2011), y se ha encontrado que tienen mayor eficiencia de forrajeo en rellenos que en ambientes naturales (Kuranchie et al. 2013).

La presencia y abundancia de aves en los rellenos sanitarios puede deberse a diferentes factores asociados a la cantidad de residuos depositados, al tipo de disposición que se realiza, al tratamiento dado a los residuos, al nivel de operación del RS, entre otros (Patton 1988; Spaans et al. 1991; Vermeer \& Irons 1991; Pons 1992; Gabrey 1997; Giaccardi et al. 1997; Lobos et al. 2011), como así también a factores paisajísticos y regionales [i.e., abundancia local y regional de las especies, ambientes adecuados de cría, alimentación, descanso y /o refugio en las cercanías de los rellenos, entre otros (Gabrey 1997)]. Las abundancias totales de aves y de palomas en los rellenos de este estudio se relacionaron en parte a la superficie de residuos expuestos, lo cual depende de la cantidad diaria de residuos que se depositan, mientras que el uso de hábitat de los distintos grupos funcionales parece depender de cada relleno. Sólo las gaviotas presentaron patrones comunes en los tres rellenos (utilizaron todos sus ambientes), y sólo en dos de ellos tendieron a seleccionar los ambientes acuáticos (en coincidencia con la presencia cercana de arroyos y lagunas). Estos resultados pueden ayudar a mejorar el tratamiento que deben darse a los residuos y a realizar un manejo diferencial de cada relleno sanitario en función del uso de hábitat que realizan las aves. La separación y reducción de la porción orgánica de los residuos y las acciones de manejo de los mismos durante la disposición final (e.g., máxima cobertura posible), como así también el manejo adecuado de los ambientes que rodean a los sitios de disposición final de residuos (i.e., modificación de los ambientes para hacerlos menos atractivos para las aves) y métodos directos de control de aves, ayudaría a disminuir sus abundancias dentro de los rellenos. Dichas medidas, además, son una forma de disminuir, o al menos de mantener estable, los niveles poblacionales actuales de algunas especies de aves oportunistas como las gaviotas y palomas (Coulson et al. 1987; Meathrel et al. 1991; Spaans \& Blokpoel 1991; Pons 1992; Giaccardi et al. 1997) disminuyendo así los potenciales efectos negativos sobre el hombre y el medio ambiente.

Agradecimientos: A R Almagro, L Bustos, M Cenizo, I Godoy, M Lucía, L Marti, L Pagano, F Richter y G Schargorodsky por su ayuda en los muestreos de campo. A F Milesi y a dos revisores anónimos por la revisión crítica de la primera versión del manuscrito. A CEAMSE por la financiación del trabajo.

\section{BIBLIOGRAFÍA}

AMERICAN SOCIETY OF CIVIL ENGINEERs. 1969. Sanitary Landfill. ASCE manual of engineering practice 39. ASCE, New York, EE.UU.

BELANT, JL. 1997. Gulls in urban environments: landscapelevel management to reduce conflict. Landscape Urban Plan., 38:245-258.

Belant, JL \& RA Dolbeer. 1993. Population status of nesting Laughing Gulls in the United States 1977-1991. Am. Birds, 47:220-224.

Belant, JL; TW Seamans; SW Gabrey \& SK ICKes. 1993. Importance of landfills to nesting Herring Gulls. The Condor, 95:817-830.

Bertellotti, M; P Yorio; G Blanco \& M Giaccardi. 2001. Use of tips by nesting Kelp Gulls at a growing colony in Patagonia. J. Field Ornithol., 72:338-348.

Brown, A; U Martínez Ortiz; M ACERbi \& J CorCUERA (eds.). 2006. La situación ambiental Argentina 2005. Fundación Vida Silvestre Argentina. Buenos Aires.

BlAnco, G. 1994. Seasonal abundance of Black Kites associated with the rubbish dump of Madrid, Spain. J. Raptor Res., 28:242-245. 
BlANCO, G. 1997. Role of refuse as food for migrant, floater and breeding Black Kites (Milvus migrant). J. Raptor Res., 31:71-76.

BlanCO, DE; R BAigún \& B LÓPEz-LANús. 2008. Grey-headed Gull in South America factsheet. Wetlands International for the Global Avian Influenza Network for Surveillance /WCS/USAID.

Blanco, DE \& M Carbonell (eds.). 2001. El Censo Neotropical de Aves Acuáticas. Los primeros 10 años: 19901999. Wetlands International, Buenos Aires, Argentina \& Ducks Unlimited, Inc. Memphis, EE.UU.

BLOKPOEL, H \& AL SPAANS. 1991. Introductory remarks: superabundance in gulls: causes, problems and solutions. Acta XX Congr. Int. Ornithol., 20:2361-2364.

Bó, NA; CA DARRIEU \& AR CAMPERI. 1995. Charadriiformes: Lariidae y Rynchopidae. En: Fauna de agua dulce de la República Argentina, Vol. 43: Aves, Fascículo 4c, PROFADU (CONICET), Museo de Cs. Naturales de La Plata, Argentina.

Butterfield, J; JC Coulson; SV Kearsey \& P Monaghan. 1983. The Herring Gull Larus argentatus as carrier of Salmonella. Journal of Hygiene, 91:429-436.

Canevari, M; P CAnevari; GR Carrizo; G Harris; JR Mata; ET AL. 1992. Nueva guía de las Aves Argentinas, Tomo II. Fundación Acindar, Buenos Aires, Argentina.

Conover, MR. 1983. Recent changes in Ring-billed Gull and California Gull populations in the Western United Sates. Wilson Bull., 95:362-383.

CONOVER, WJ. 1999. Practical nonparametric statistics. John Wiley \& Sons Inc., New York.

Coulson, JC; J Butterfield; N Duncan \& C Thomas. 1987. Use of refuse tips by adult British Herring Gulls Larus Argentatus during the week. J. Appl. Ecol., 24:789-800.

DONÁZAR, J. 1992. Muladares y basureros en la biología y conservación de las aves en España. Ardeola, 39:29-40.

Drury, WH. 1973. Population changes in New England seabirds. Bird Banding, 44:267-313.

GABREY, SW. 1997. Bird and small mammal abundance at four types of waste-management facilities in northeast Ohio. Landscape Urban Plan., 37:223-233.

Giaccardi, M; P Yorio \& ME Lizurume. 1997. Patrones estacionales de abundancia de la Gaviota Cocinera (Larus dominicanus) en un basural patagónico y sus relaciones con el manejo de residuos urbanos y pesqueros. Ornitol. Neotrop., 8:77-84.

Giaccardi, M \& P Yorio. 2004. Temporal patterns of abundance and waste use by Kelp Gulls (Larus dominicanus) at an urban and fishery waste site in northern coastal Patagonia, Argentina. Ornitol. Neotrop., 15:93-102.

HARRIS, MP. 1970. Rates and causes of increases of some British gull populations. Bird Study, 17:325-335.

Horton, N; T Brough \& JBA Rochard. 1983. The importance of refuse tips to gulls wintering in an inland area of southeast England. J. Appl. Ecol., 20:751-765.

Houston, DC. 1994. New World vultures. Pp. 24-41 in: del Hoyo, J; A Elliott \& J Sargatal (eds.). Handbook of the birds of the world. Vol. 2: New World vultures to Guinea Fowl. Lynx Editions, Barcelona, Spain.

HuNT, GL JR. 1972. Influence of food distribution and human disturbance on the reproductive success of Herring Gulls. Ecology, 53:1051-1061.

Kadlec, JA \& WH Drury. 1968. Structure of the New England Herring Gull population. Ecology, 49:644-676.

KiHLMAN, J \& L LARSSON. 1974. On the importance of refuse dumps as a food source for wintering Herring Gulls Larus argentatus Pont. Ornis Scand., 5:63-70.

Kuranchie, A; K Rosina; AK Daniel \& LH Holbech. 2013. Foraging activities, success and efficiency of Cattle Egrets (Bubulcus ibis) in three habitat types in the Greater Accra Region of Ghana. J. Biol. Food Sci. Res., 2:45-50.

Lobos, G; P Bobadilla; A Alzamora \& RF Thompson. 2011. Patrón de actividad y abundancia de aves en un relleno sanitario de Chile central. Rev. Chil. Hist. Nat., 84:107-113.

López-Lanús, B \& DE Blanco (eds.). 2005. El Censo Neotropical de Aves Acuáticas 2004. Global Series No 17. Wetlands International, Buenos Aires, Argentina.

Meathrel, CE; JA Mills \& RD Wooller. 1991. The Silver Gull in Australia and New Zealand. Acta XX Congr. Int. Ornithol., 20:2390-2395.

Monaghan, P; BC SHedden; K Ensor; CR Fricker \& RWA GIRWOOD. 1985. Salmonella carriage by Herring Gulls in the Clyde area of Scotrland in relation to their feeding ecology. J. Appl. Ecol., 22:669-680.

NAROSKy, T \& A Di GiÁcomo. 1993. Las aves de la provincia de Buenos Aires: distribución y estatus. Asoc. Ornitológica del Plata, Vázquez Mazzini Ed. and L.O.L.A. Buenos Aires, Argentina.

Patton, SR. 1988. Abundance of gulls at Tampa Bay landfills. Wilson Bull., 100:431-442.

Pomeroy, DE. 1975. Birds as scavengers of refuse in Uganda. Ibis, 117:69-91.

Pons, JM. 1992. Effects of changes in the availability of human refuse on breeding parameters in a Herring Gull Larus argentatus population in Brittany, France. Ardea, 80:143-150.

QuinN, GP \& MJ KeOugh. 2002. Experimental design and data analysis for biologists. Cambridge University Press, New York.

Silva Rodríguez, MP; M Favero; MP Berón; RM Jelicich \& L MAuco. 2005. Ecología y conservación de aves marinas que utilizan el litoral bonaerense como área de invernada. El Hornero, 20:111-130.

SoDHI, NS. 2002. Competition in the air: birds versus aircraft. $A u k, 119: 587-595$.

SpaANS, AL \& H BlokPOEl. 1991. Concluding remarks: superabundance in gulls: causes, problems and solutions. Acta XX Congr. Int. Ornithol., 20:2396-2398.

Spanns, Al; JC Coulson; P Migot; P Monaghan; J Pruter; ET AL. 1991. The Herring Gull in north-west Europe. Acta XX Congr. Int. Ornithol., 20:2365-2371.

VermeER, K \& DB Irons. 1991. The Glaucous-Winged Gull on the pacific coast of North America. Acta XX Congr. Int. Ornithol., 20:2378-2383.

White, CM; PD Olsen \& LF KIFF. 1994. Falcons. Pp. 216-275 en: del Hoyo, J; A Elliott \& J Sargatal (eds.). Handbook of the birds of the world. Vol. 2: New World vultures to Guinea Fowl. Lynx Editions, Barcelona, Spain.

Yorio, P; E Frere; P GANDini \& M Giaccardi. 1996. Uso de basurales urbanos por gaviotas: magnitud del problema y metodologías para su evaluación. Informe Técnico del Plan de Manejo Integrado de la Zona Costera Patagónica, Fundación Patagonia Natural (Puerto Madryn, Argentina) 22:1-22.

YORIO, P \& M GIACCARDI. 2002. Urban and fishery waste tips as food sources for birds in northern coastal Patagonia, Argentina. Ornitol. Neotrop., 13:283-292.

Yorio, P; M Bertellotti \& P García Borboroglu. 2005. Estado poblacional y de conservación de gaviotas que se reproducen en el litoral marítimo argentino. El Hornero, 20:53-74. 